Ibrahim Güven

Institute of Mechanics,

Ruhr-University Bochum,

Universitätsstr. 150,

Bochum D-44 801, Germany;

Multi-Scale Mechanics/MESA+, ET,

University of Twente,

Drienerlolaan 5,

Enschede 7522 NB, The Netherlands

e-mail: ibrahim.gueven@gmx.de

Stefan Luding

Multi-Scale Mechanics/MESA+, ET,

University of Twente,

Drienerlolaan 5

Enschede 7522 NB, The Netherlands

e-mail: s.luding@utwente.n

Holger Steeb

Institute of Applied Mechanics (CE),

University of Stuttgart, Pfaffenwaldring 7 ,

Stuttgart D-70 569, Germany;

Stuttgart Research Center for Simulation Technology, Pfaffenwaldring $5 \mathrm{a}$

Stuttgart D-70569, Germany e-mail: steeb@ist.uni-stuttgart.de

\section{Incoherent Waves in Fluid- Saturated Sintered Granular Systems: Scattering Phenomena}

The incoherent transport of ultrasound waves in water-saturated sintered glass bead packings is experimentally investigated. The spectral energy density of scattered highfrequency waves is explained by a diffusion wave equation. Immersion broadband transducers with central frequencies of $1 \mathrm{MHz}$ are positioned at a distance of $73 \mathrm{~mm}$ to the porous sample. The diffusion coefficient and quality factor are predicted from a diffusion approximation of the time-dependent intensity curve to the ensemble-averaged measurement data. From the diffusion coefficient, we deduce a mean-free path for scattering events at $l^{*}=0.87 \pm 0.03 \mathrm{~mm}$ close to the range of particle diameters of the samples $\left(1.0<d_{p}<1.2 \mathrm{~mm}\right)$. Results are in good agreement with observations from Jia (2004, "Codalike Multiple Scattering of Elastic Waves in Dense Granular Media," Phys. Rev. Lett., 93(15), p. 154303) observed for nonsintered and consolidated bead packings $\left(0.6<d_{p}<0.8 \mathrm{~mm}\right)$. The low-quality factor $Q=190 \pm 10$ indicates a high amount of intrinsic damping of the scattered waves although water was used as saturating and coupling fluid. [DOI: 10.1115/1.4037701]

\section{Introduction}

Porous and granular media are present in many industrial applications as well as in our daily life, for instance, in pharmaceutics or food-processing like tablets or nuts. Fluid-filled porous media are ubiquitous in natural and engineered systems. Examples are the water storage in soil and the flow of fluids like oil, gas, and water through porous petroleum reservoirs or through organic materials such as porous bone structures filled with bone marrow.

Ultrasound as a noninvasive and effective testing method is commonly used for characterization of such materials, cf. Refs. [1-6]. It enables us to determine essential micro- and macrostructural parameters of the medium, which can finally be used in numerical simulations to model the acoustical response. Therefore, understanding of the propagation of acoustic waves in porous and granular media is of relevance for various applications in geosciences and engineering.

In classical seismics, i.e., low-frequency applications, elastic or visco-elastic models describe well the inherent wave propagation processes for consolidated granular media. For fluid-saturated porous materials, Biot $[7,8]$ proposed dynamic poroelastic equations which are extending the elastic Lamé-Navier equations and predict the existence of a second compressional wave (Biot's slow wave). Even in the high-frequency extension of Ref. [8], where a deviation of the parabolic or Poiseuille-type fluid velocity distribution in pore channels is taken into account, Biot's model is based on a continuum approach, i.e., the microstructure of the porous skeleton is significantly smaller than the wavelength of the incident wave. If the wavelength of the acoustic wave approaches the order of the characteristic microstructures of the granular medium (e.g., $\lambda \approx d_{50}$ ), such continuum models must fail and further physical processes like wave scattering occur, cf. Refs. $[9,10]$. Determining the amount of effective attenuation caused by scattering by experimental investigations is necessary to develop more advanced models allowing to predict these effects.

Contributed by the Technical Committee on Vibration and Sound of ASME for publication in the Journal OF VIBRATION AND ACOUSTICS. Manuscript received December 28, 2016; final manuscript received August 5, 2017; published online October 4, 2017. Assoc. Editor: Matthew Brake.
Sintered glass bead packings are interesting synthetic porous systems for studying wave propagation as the characteristic length scales of the microstructure (particle diameter $d_{p}$ ) can be fixed before the sinter process and adapted to the wavelength under investigation. Still, such particles are far from being ideally monodisperse. After sintering, the packings are even more heterogeneous due to temperature variations and inhomogeneities in the production process. The heterogeneities due to the random distribution of polydisperse glass beads in the solid matrix can lead to strong scattering of ultrasound waves at wavelengths in the order of the characteristic diameter of the glass beads $\left(\lambda \approx d_{p}\right)$. Scattering of ultrasonic waves is further enhanced through microcracks resulting from rapid cooling processes during sintering. Therefore, the use of relatively high-frequency ultrasound waves (in the megahertz range) can provide useful information about the microstructure of strongly disordered inhomogeneous materials similar to mechanisms in various geological materials.

Previous investigations showed that in addition to a classical wave propagation process of a coherent pulse, a diffusion approximation of the scattered part of the wave signal can be successfully applied under the conditions of strong multiple scattering in randomly distributed dry and wet glass bead packings, cf. Ref. [11]. Griffiths et al. [12] used diffusive ultrasound to investigate nonconsolidated granular materials in vacuum or saturated with different kinds of gases and determined the frequency-dependent diffusion parameters. However, in their investigations, the scattering medium was directly excited using shear-mode activating bender elements.

In this study, diffusive ultrasound experiments are extended toward fully fluid-saturated sintered glass bead systems, where water is used as coupling medium and pore fluid. The applicability of the diffusion model, in which wave interference is completely ignored, is tested on the incoherent wave part of the received ultrasound signal. Using the diffusion extension of the classical wave equation, we are able to determine the diffusion coefficient and a relatively low-quality factor in the disordered sample.

In Sec. 2, the experimental procedure and setup for the performed ultrasound experiments is briefly described. In Sec. 3, the diffusive wave transport of ultrasound is briefly introduced and 


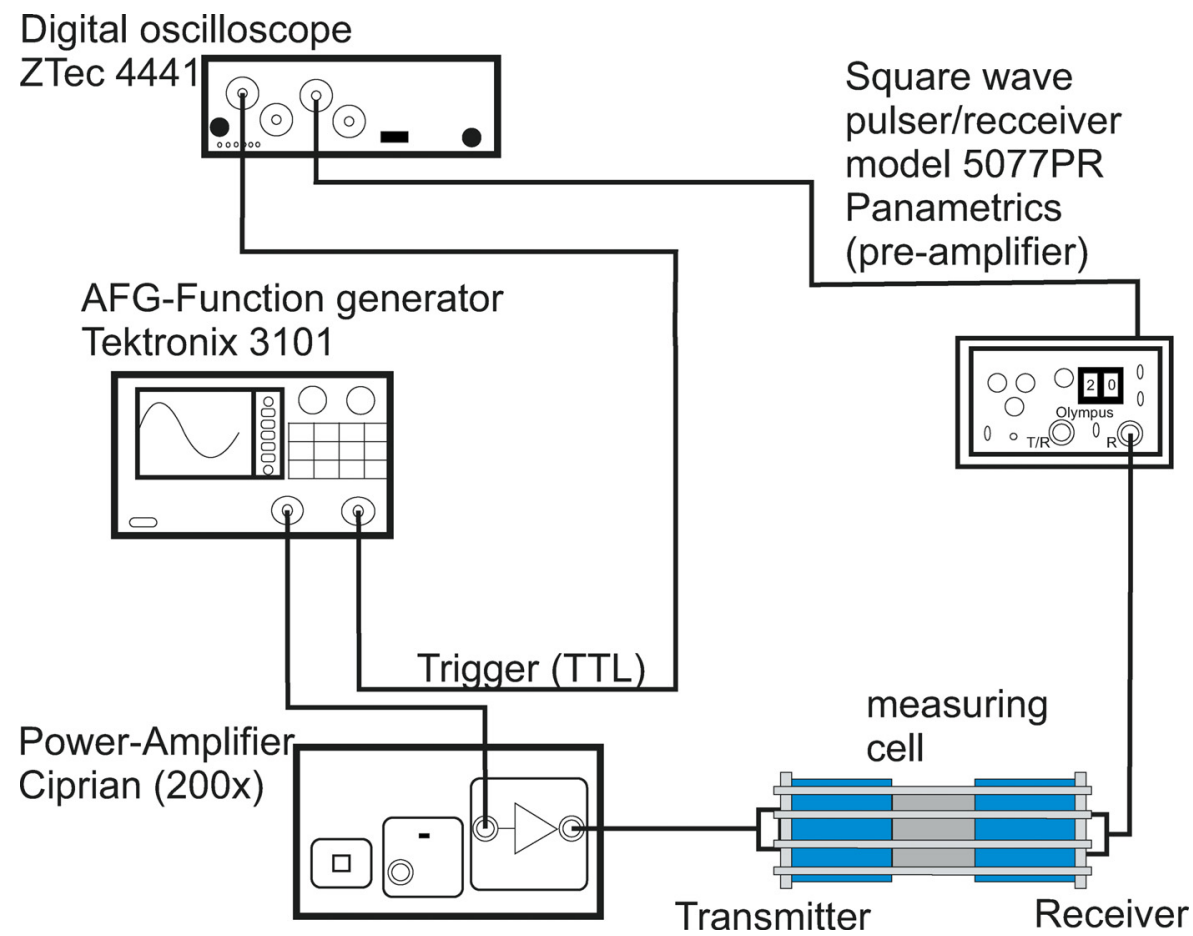

Fig. 1 Experimental setup according to the transmission method, where the square wave pulser of Olympus (model 5077PR) is used as pre-amplifier

approximated to the results obtained from the measurement of a water-saturated sample with glass bead sizes around $1.1 \mathrm{~mm}$. The observations and results are finally discussed and concluded in Sec. 4.

\section{Experiments}

The experimental setup is a classical configuration to carry out immersion ultrasound measurements according to the transmission technique, cf. Fig. 1. The ultrasound experiments are performed in an in-house developed measuring cell made of PMMA. A detailed description of the cell is given in Ref. [13]. The electrical lowvoltage input signal is generated by an arbitrary function generator (Tektronix AFG 3101) and amplified to $\pm 400 \mathrm{~V}$ by a linear power amplifier (Ciprian US-TXP-3). The received low-voltage time signal is pre-amplified (Panametrics 5077PR), displayed, and digitized with a high-resolution digitizer (ZTec 4441, $300 \mathrm{MHz}$ bandwidth, sample rate 800 MSamples/s, vertical resolution 14 bit).

Two immersion broadband transducers with central frequencies of $1 \mathrm{MHz}$ (Olympus-Panametrics NDT, V303-SU) are placed in the water reservoir at the front and back of the porous sample. The immersion broadband transducers are not in direct contact with the investigated sample, see Fig. 2. The distance between the transducers and the porous sample is fixed at $73 \pm 0.4 \mathrm{~mm}$, cf. Fig. 2 .

Prior to the ultrasound investigations, the cell, including the porous sample, is rinsed out with carbon dioxide and filled with de-aired and de-ionized water, in order to achieve an optimal

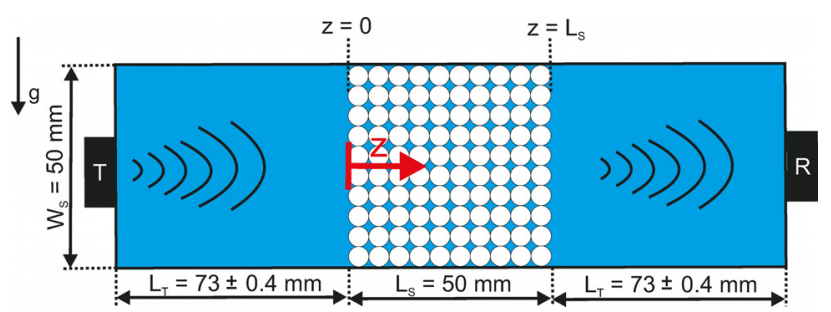

Fig. 2 Simplified sketch of the measuring cell with length specifications used for ultrasound measurements saturation of the sample and to minimize the chance for remaining of air bubbles. A ten-cycle-sinus burst excitation at a frequency of $0.9 \mathrm{MHz}$ is applied to the piezoelectric P-wave source transducer. The narrow band excitation corresponds to the product of the acoustic wave number and glass bead diameter $k d_{p}=\omega d_{p} / c_{P 1} \approx$ 2.2 with $c_{P 1}=2872.5 \mathrm{~m} / \mathrm{s}$ as the bulk velocity and $d_{p}=1.1 \mathrm{~mm}$ as the (average) glass bead diameter forming the solid skeleton. Note that the wave velocity was determined from the time of flight difference $(\triangle \mathrm{TOF})$ of the ultrasound signals with (TOF) and without $\left(\mathrm{TOF}_{\mathrm{ref}}\right)$ positioning a porous sample according to

$$
c_{P 1}=\frac{1}{\frac{1}{c_{\mathrm{ref}}}+\frac{\Delta \mathrm{TOF}}{L_{S}}}
$$

where $\quad \Delta \mathrm{TOF}=\mathrm{TOF}-\mathrm{TOF}_{\text {ref }} \quad$ with $\quad \mathrm{TOF}=115.7 \mu \mathrm{s} \quad$ and $\mathrm{TOF}_{\text {ref }}=132 \mu \mathrm{s}$. The reference velocity in water at $22^{\circ} \mathrm{C}$ and the sample length was determined at $c_{\text {ref }}=1485 \mathrm{~m} / \mathrm{s}$ and $L_{S}=50 \mathrm{~mm}$. The wave velocity of the coherent impulse corresponds to the velocity of the fast P1-wave and results mainly from the inertial coupling of both phases as result of the tortuosity of the pore channels, cf. Ref. [8].

In these high-frequency ranges, a strong ultrasound scattering of the investigated medium is expected, where the wavelength of the ultrasound wave is only about $\lambda=3.2 \mathrm{~mm}$ and thus in the order of the particle diameter $d_{p}$, cf. Ref. [9]. Figure 3 shows a typical received time signal of a water-saturated sintered glass bead sample with bead diameters between 1.0 and $1.2 \mathrm{~mm}$.

The investigated cylindrical sample (with diameter and length $50 \mathrm{~mm}$ ) has an open pore structure and effective values of $\phi=$ $31.87 \%$ for the porosity and $K_{z}^{s}=3.10 \times 10^{-10} \mathrm{~m}^{2}$ for the intrinsic permeability. The porosity was determined after sintering from the bulk density of the porous sample and the specific density of the beads. The intrinsic permeability was determined in stationary permeability experiments, cf. Refs. $[13,14]$. Figure 4 shows the probability distribution for the equivalent particle diameter (solid line) with the corresponding cumulative curve (dashed line) determined from X-ray computed tomography (XRCT) scans after sintering. The data shown in Fig. 4 are obtained from a subset, which contained approximately 14,000 particles, see Fig. 5. The equivalent 


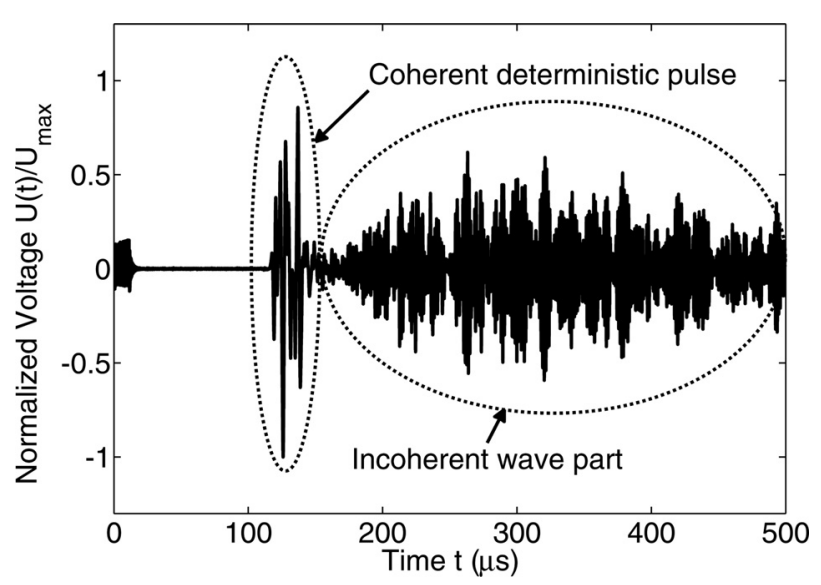

Fig. 3 Received time signal in raw state

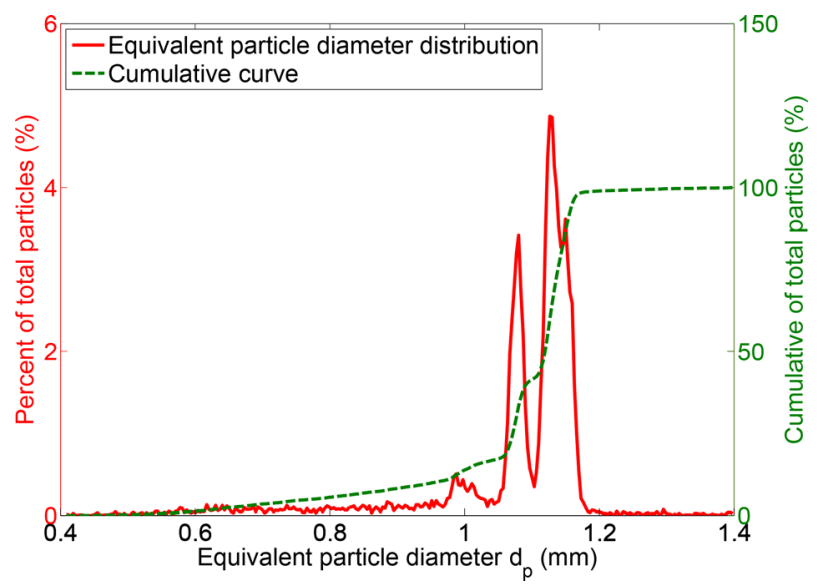

Fig. 4 Probability distribution of equivalent particle diameter (solid line) and corresponding cumulative curve (dashed line) determined from XRCT scans after sintering

particle diameters are determined from the segmented voxel-based volumes $V_{p}$ of the particles according to $d_{p}=\sqrt[3]{6 V_{p} / \pi}$.

The time signal in Fig. 3 is basically composed of a coherent pulse arriving at the leading edge and an incoherent part mainly composed of specklelike multiple-scattered waves, where only the latter are highly sensitive to the underlying microstructure of the investigated sample.

For the diffusion approximation, only the incoherent wave part is considered. The dominant energy component of the transmitted wave is at a high frequency, which has a multiply scattered behavior approaching a diffusive regime $[9,11,15]$. In the diffusive regime, shear waves, which arise from persistent local wave mode conversions of $\mathrm{P}$-waves to $\mathrm{S}$-waves within the porous sample, are believed to dominate the scattered wave field $[11,16]$.

Two distinct ultrasound scattering mechanisms may occur in such fluid-saturated sintered granular systems. The first travels through the solid phase (sintered glass beads) and the second transmits along the fluid phase (water). The data shown in Fig. 3 correspond likely to the first kind as in Refs. [11], [12], [17], and [18] and not to the second as in Refs. [19-21].

Weaver and Sachse [19], who have investigated the sound transmission through glass bead slurries, anticipated that the acoustic signals mainly travel through the fluid and scattered from the bead surfaces. Thus, waves do not propagate to any significant degree from bead to bead due to weak stiffness between the particles, different from this study. The existing strong sintering contacts between the particles have resulted in a significant increase of the stiffness between the particles. This is also confirmed by

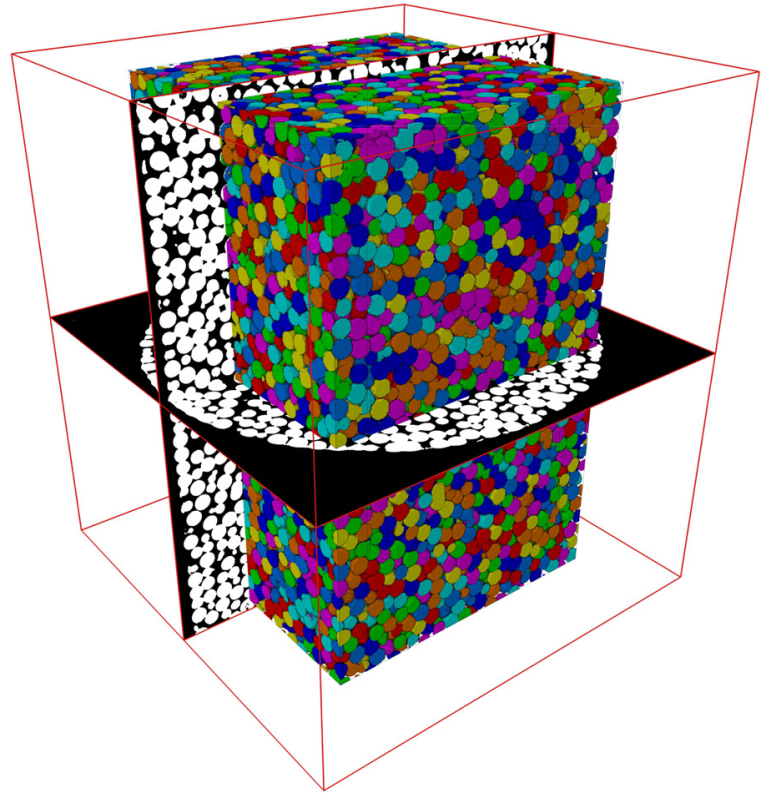

Fig. 5 Investigated subset from XRCT scan with 14,053 particles (voxel resolution $16 \mu \mathrm{m}$ ) used for the determination of the equivalent particle diameter distribution shown in Fig. 4. The bounding box indicates the entire scanned region. The cuboidshaped subset has the dimensions of $1940 \times 1965 \times 2200$ voxel $^{3}$ $\left(31.04 \times 31.44 \times 35.20 \mathrm{~mm}^{3}\right)$.

the fact that the amplitude (energy) of coherent deterministic pulse decreased in relation to the amplitude (energy) of scattered waves with increasing particle diameter and sintering duration (samples with lower porosity), which indicates that the scattered ultrasound energy is transported mainly through the solid, cf. Refs. $[10,13]$. The scattering process is additionally enhanced by the existing local porosity and intrinsic permeability gradients along the propagation direction, which were determined by XRCT analysis and used in lattice Boltzmann simulations, cf. Refs. $[13,14]$.

Similar observations were also made by Jia et al. [17], who have investigated dry and wet glass bead packings under external loading and found that no ultrasound signal was detected at vanishing external load. Moreover, they could show that the intensity as well as the amount of scattered waves decreased when the beads were wetted with oil. These tests indicate that the scattered ultrasound waves propagate from one grain to its neighbors only through their mutual contacts and not via the saturating fluid.

Nonlinear multiple wave scattering effects, which result from microscopic small rearrangements of the particles as result of weak contact between the particles and influence the coda part of the received ultrasound signals as described in unconsolidated media in Refs. [22-24], are excluded in the performed ultrasound experiments, since sintered granular systems with strong interparticle contacts are considered, in which it is impossible that particles slide or slip under the action of varying small amplitude strain waves. The amplitudes are much smaller than necessary to destroy or influence the packing structure.

Using temporal windows for the calculations of the power spectra of the coherent and incoherent parts reveals that the frequency content of the coherent wave part is considerably lower than that of the incoherent part, cf. Fig. 6. The irregular high-frequency spectrum of the incoherent part as well as the low-frequency spectrum of the coherent part was confirmed by Jia et al. [17].

However, from the comparison between the power spectra of the total received signal and the coherent part, a clear visible difference becomes apparent, cf. Fig. 6. The spectrum of the coherent pulse does not coincide with the low-frequency part of the total power spectrum. The incoherent part contains also a 


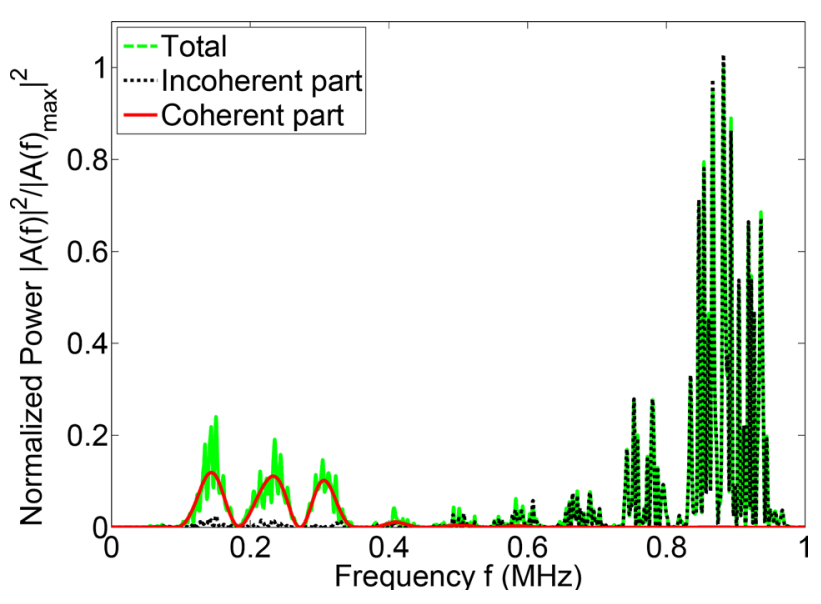

Fig. 6 Corresponding power spectra of the raw signal

low-frequency wave part, which, for instance, results from the back reflection of the P1-wave from the sample or from the occurrence of the so-called slow P2-wave $[7,8]$. These waves commonly have the same frequency content as the coherent pulse [25]. Therefore, for an optimal separation of the high-frequency incoherent part from the remaining part of the received time signal, a high-pass filter is applied to the raw signal. Note that the classical S-wave propagation according to Biot's theory is excluded in the performed experiments, since the P-wave transmission normal to the surface area of the porous sample is considered. The $\mathrm{P}$ - and $\mathrm{S}$-waves generated from local wave mode conversions in the scattered wave field have much higher frequencies and do not influence the low-frequency spectrum as shown in Fig. 6. Reflections at the edges of the measuring cell are also excluded for the considered time period due to the small opening angle of the ultrasound cone starting from the transmitter.

Moreover, it can be seen in Fig. 6 that the dominant frequency range of the coherent wave part lies approximately between 0.16 and $0.37 \mathrm{MHz}$, which is certainly lower than the central frequency of the broadband excitation signal $(0.9 \mathrm{MHz})$. This indicates the well-known low-pass filter behavior of a porous and granular medium and is consistent with results obtained from previous studies, cf. Refs. [26-28].

As can be seen in Fig. 6, the power of the incoherent scattered wave part is certainly higher than the power of the coherent part, which indicates that the most energy is transported in the incoherent diffusive wave part. The power ratio between the coherent and incoherent wave part can change depending on the frequency of the excitation signal, cf. Refs. $[10,13]$.

Figure 7 shows the high-pass-filtered time signal, where the cutoff frequency has been chosen at $0.7 \mathrm{MHz}$ at which the lowfrequency coherent pulse at the leading edge disappears. Note that the typical scattered sound wave field presented in Fig. 7 could only be observed in our experiments for a narrow frequency range of $0.9-1.1 \mathrm{MHz}$, where the wavelengths are in the order of the particle sizes and have a high signal-to-noise ratio. At very high frequencies $(>1.2 \mathrm{MHz})$, the received signals are attenuated more strongly, which is strongly related to our setup. The corresponding power spectrum of the high-pass-filtered signal is shown in Fig. 8, i.e., the low-frequency part $(f<0.65 \mathrm{MHz})$ is mostly filtered out.

\section{Diffusive Wave Propagation}

From the time signal, depicted in Fig. 3, we can see that most of the energy transport through the water-saturated sintered glass bead sample is caused by scattered waves occurring in the incoherent part of the received time signals. Although it is challenging to describe the multiple scattering of classical waves, significant progress has been achieved through the use of the diffusive wave

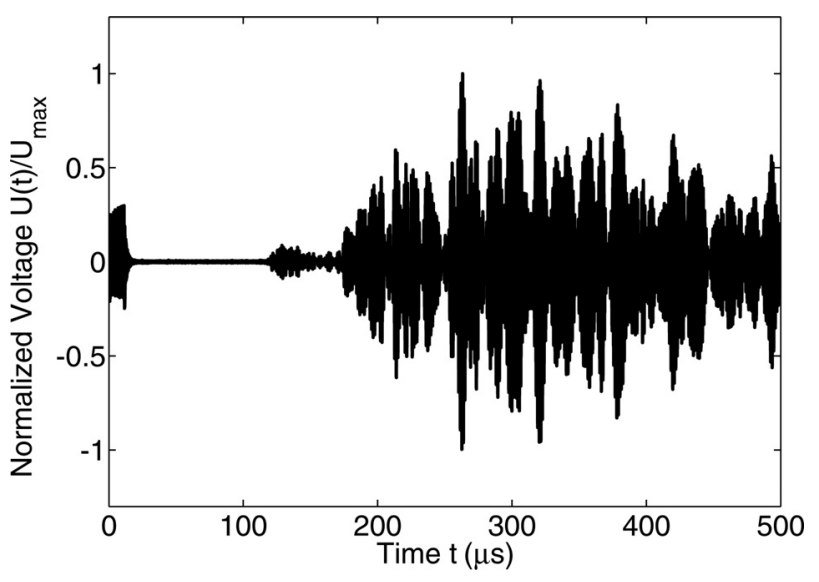

Fig. 7 Received time signal after high-pass filtering. The cutoff frequency is chosen at $0.7 \mathrm{MHz}$.

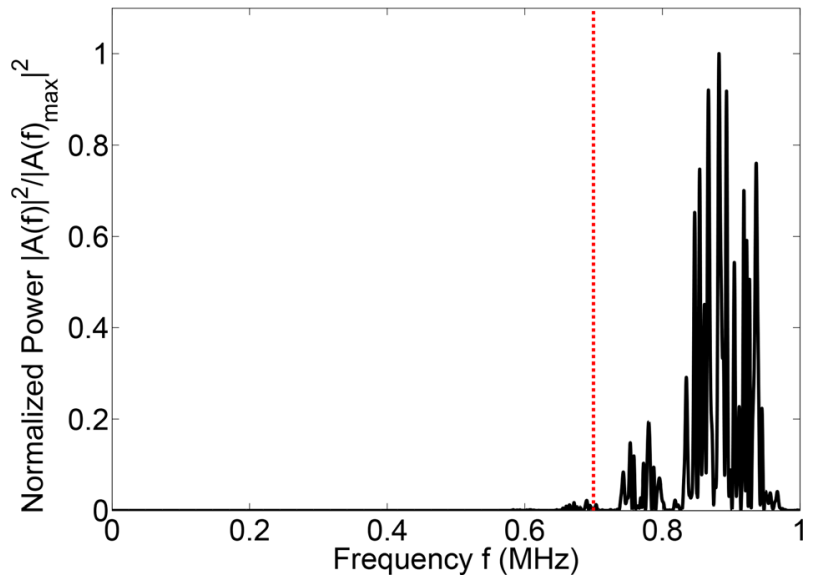

Fig. 8 Corresponding power spectra of the high-pass-filtered high-frequency incoherent part. The vertical dotted line indicates the selected cutoff frequency at $0.7 \mathrm{MHz}$.

approximation [19,29-34]. In the diffusive model of multiplescattered waves, the averaged wave intensity is treated as a (isotropic) random walk process. The phase information is not considered and the wave propagation is described by a single diffusion coefficient as

$$
D=\frac{1}{3} c_{e} l^{*}
$$

where $l^{*}$ represents the transport mean-free path, which describes the mean distance the ultrasound wave travels before its propagation direction is randomized by a scattering "event" [11]. $c_{e}$ is the averaged ballistic velocity, at which the energy of the scattered waves is transported through the porous sample-water system.

The diffusion method is based on the assumption that the spectral energy density of the scattered ultrasonic wave field is well described by a diffusion equation. The diffusive wave field is temporally and spatially incoherent with the incident wave and the field variables are assumed to be random, see Ref. [35]. According to Deroo et al. [35] and Weaver et al. [36], the diffusion equation that describes the time evolution of the spectral energy density $\bar{E}$ : $=\langle E(z, t, f)\rangle$ of an ultrasonic wave field can be formulated as

$$
\frac{\partial \bar{E}}{\partial t}-D \operatorname{divgrad} \bar{E}=P-\sigma \bar{E}
$$

where $P(z, t, f)$ is the spectral energy density source term (forcing condition) and $\sigma(f)$ is the dissipation rate. The diffusion 
coefficient $D$, which depends also on the frequency excitation signal, is very sensitive to the microstructure, but is assumed to be isotropic, i.e., independent from direction.

Fast spatial variations in amplitude and phase, which are typical for a diffusive wave field, converge to zero when they are ensemble-averaged over various configurations $[35,37,38]$. This property of waves is known as phase cancellation. It should be noticed that the energy in the coherent wave part is transferred to the incoherent wave part consisting of (multiple) scattered waves with increasing frequency of the incident wave, c.f. Refs. [10,13]. No energy is lost in scattering and scattering has no contribution to the dissipation rate $\sigma$ [35]. A higher diffusion coefficient $D$ results in faster diffusion of energy transported in the multiplescattered waves, whereas an increasing scattering probability is associated with slowing down of the diffusive process and decreasing the diffusion coefficient. As highlighted by Page et al. [39], the energy density measured on the same axis as the source at the distance $z=L_{S}$ can be approximated by a one-dimensional plane wave solution at various frequencies in the scattering sample. Under the assumption that the dissipation rate cell walls are perfectly reflecting at $z=0$ and $z=L_{S}$, Jia [11] has proposed a simple solution for the time-dependent intensity profile $I(t)$ based on a Fourier-series approach [40]

$$
\begin{aligned}
I(t) & =-\left.D \frac{\partial E}{\partial z}\right|_{z=0} ^{z=L_{S}} \\
& =\frac{c_{e} E_{0}}{2 L_{S}} e^{-t / \tau_{a}} \sum_{n=0}^{N} \frac{(-1)^{n}}{\delta_{n}} \cos \left(\frac{n \pi l^{*}}{L_{S}}\right) e^{-D(n \pi)^{2} t / L_{S}^{2}}
\end{aligned}
$$

where $E_{0}$ is the energy of the transmitted scattered waves.

$\delta_{n}=2$ for $n=0$, otherwise $\delta_{n}=1 . \tau_{a}$ describes the effective inelastic absorption time and can be expressed in terms of the "coarse-grained" effective quality factor of the scattered waves as

$$
\tau_{a}=\frac{Q}{2 \pi f}
$$

Ultrasound experiments can be used to measure the diffusion coefficient and the quality factor from the time evolution of the transmitted high-frequency scattered waves. In this aspect, the measured transmitted acoustic intensity is determined by ensemble-averaging the square of the envelope of the scattered sound field, which is represented in the incoherent wave part of the received time signal $[12,29,38,41]$, see Figs. 3 and 7.

Figure 9 shows a typical result of the evaluation of scattered ultrasound waves in a water-saturated glass bead sample. For a better display and comparison of the experimental and theoretical curves, the vertical axis gives the normalized intensity values and is logarithmic, which explains the negative sign of the values on the vertical axis.

The measured intensity curve results from the averaging of ten independent ultrasound experiments and is compared with the theoretical intensity curve, represented by the solid line and determined from the solution of the diffusion equation given in Eq. (4). The ultrasound measurements are obtained from five different samples with the same glass bead diameters and sintering treatment. For each investigated sample, the transmitter and receiver have been replaced. The averaged intensity curve of the scattered waves from the ultrasound measurements is determined by squaring the envelope of the filtered waveforms. To ensure a welldefined ultrasonic frequency, a sinus-burst-signal with ten cycles is used. The frequency of the generated quasi-monochromatic wave has been chosen at $0.9 \mathrm{MHz}$. The diffusion approximation, which is normalized to the peak of the intensity, is calculated by using the diffusion coefficient and quality factor as fit parameters. For the water-saturated sintered sample with bead diameters between 1.0 and $1.2 \mathrm{~mm}$, the best fit is obtained with the set of parameters $D=0.83 \pm 0.03 \mathrm{~m}^{2} / \mathrm{s}$ and $Q=190 \pm 10$, where the

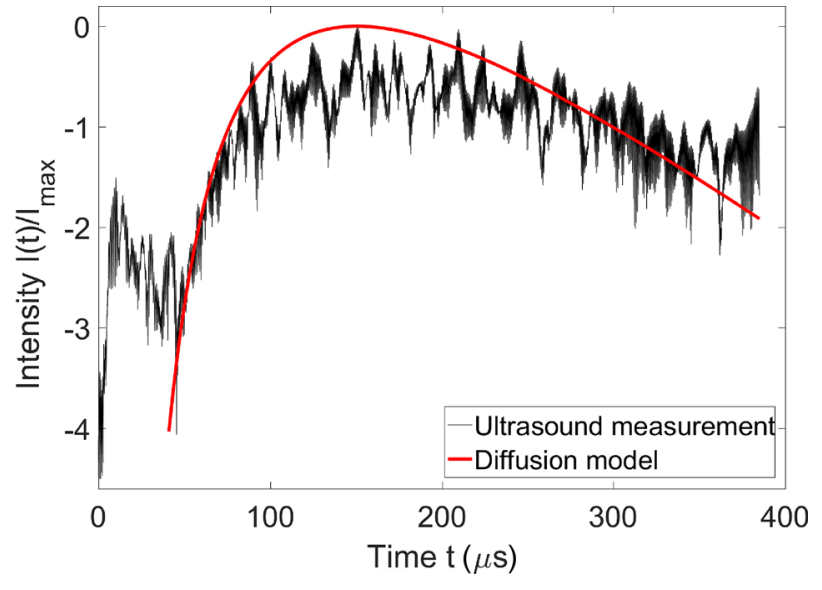

Fig. 9 Comparison of normalized intensities obtained from ultrasound measurements and the related diffusion model. The measured averaged intensity curve is determined from ten independent experimental measurements. The piezoelectric acoustic transmitter is excited with a ten-cycle-sinus burst at $0.9 \mathrm{MHz}$. The diffusion coefficient and the effective quality factor are determined fitting the diffusion model to the measured averaged intensity profile at $D=0.83 \pm 0.03 \mathrm{~m}^{2} / \mathrm{s}$ and $Q=190 \pm 10$.

number of speckles is chosen at $N=150$. The number of speckles $N$ does not influence the fit parameters, if it is selected large enough $(N>8)$. Note that for the fit of Eq. (4), a new temporal axis is defined with $t=\mathrm{TOF}=0$ as the beginning of the penetration of the ultrasound wave into the porous sample. In this way, reference is made only to events, which have taken place within the porous sintered sample, and the experimental configuration is adapted to the used Eq. (4).

In accordance with Jia et al. [11,17], the energy transport velocity introduced in Eq. (2) is identified with the determined coherent wave as $c_{e}=c_{P 1}=2872.5 \mathrm{~m} / \mathrm{s}$. Note that this assumption provides also an appropriate fit to the measurement data. The fact that the (multiply) scattered waves arrive at certainly later times than the coherent wave impulse is due to the fact that the (multiply) scattered waves are generated at later times after the coherent wave impulse propagated through the water-saturated porous sample. Further input parameters used to predict the time-dependent intensity curve and important results are summarized in Table 1.

Jia [11] has determined the diffusion coefficient and quality factor for nonsintered, dry glass beads with $d_{p}=0.6-0.8 \mathrm{~mm}$ at $0.13 \pm 0.01 \mathrm{~m}^{2} / \mathrm{s}$ and $200 \pm 10$, which is rather close to our model-fit results from our experiments.

Differences in the results are caused by different particle sizes, polydispersities, and especially, the fact that the investigated samples in this study were sintered and water was used as saturating and coupling fluid.

Since the scattered sound waves mainly propagate through the solid phase, the determined $Q$-value is highly affected by the

Table 1 Input parameters for the diffusion model and results obtained from the fit of the theoretical time-dependent intensity curve to measurement data

\begin{tabular}{lcc}
\hline \hline Parameter & Unit & \\
\hline Sample length, $L_{S}$ & $(\mathrm{~mm})$ & 50 \\
Energy transport velocity, $c_{e}$ & $(\mathrm{~m} / \mathrm{s})$ & 2872.5 \\
Frequency, $f$ & $(\mathrm{MHz})$ & 0.9 \\
Number of speckles, $N$ & & 150 \\
Diffusion coefficient, $D$ & $\left(\mathrm{~m}^{2} / \mathrm{s}\right)$ & $0.83 \pm 0.03$ \\
Quality factor, $Q$ & & $190 \pm 10$ \\
Transport mean-free path, $l^{*}$ & $(\mathrm{~mm})$ & $0.87 \pm 0.03$ \\
Glass bead diameter, $d_{p}$ & $(\mathrm{~mm})$ & $1.0-1.2$ \\
\hline \hline
\end{tabular}




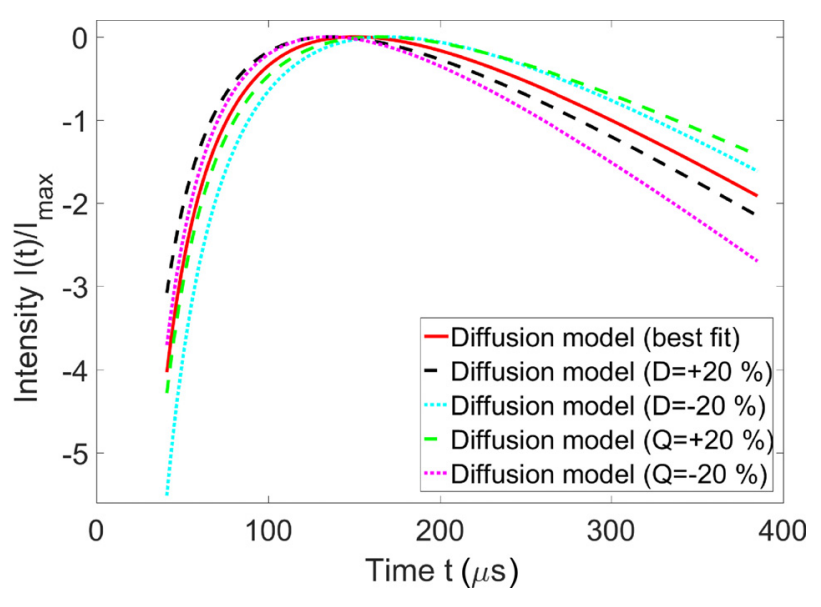

Fig. 10 Diffusion model for different parameters of the diffusion coefficient and quality factor. The best fit for the normalized intensity curve from the ultrasound measurement, shown in Fig. 9, is represented by the solid line. The remaining intensity curves refer to different $D$ and $Q$ values with deviations of $\pm 20 \%$ from the best-fit intensity curve.

existing sinter contacts between the particles and the inhomogeneities along the propagation direction (due to sintering procedure). But also dissipation mechanisms due to the presence of water in the pore space, which mainly result from inertial than from viscous dissipation, can influence the quality factor. Attenuation effects from persistent local wave conversions (between S- and P-waves) in the scattered wave field as well as internal friction within the glass beads can also contribute to the determined $Q$-value.

The transport mean-free path of the ultrasound wave is determined according to Eq. (2) at $0.87 \pm 0.03 \mathrm{~mm}$. This means that the ultrasound wave changes its direction and is being randomized approximately every $0.87 \pm 0.03 \mathrm{~mm}$ in the porous sample, which is close to the diameter of the particles.

Figure 10 demonstrates the sensitivity of the used diffusion model toward different values of the diffusion coefficient and the quality factor. It shows the time-dependent normalized intensity profiles with deviations of $\pm 20 \%$ for the diffusion coefficient and quality factor from the ideal intensity curve represented by the solid line and used to approximate the ultrasound measurements, cf. Fig. 9. It can be seen that the intensity curve increases with increasing diffusion coefficient, whereas the intensity curve decays slower for increasing quality factor due to the lower effective attenuation properties of the scattering medium.

The initial rise of the theoretical curves is determined by the diffusion coefficient, while the declining of the curves at later times $(t>180 \mu \mathrm{s})$ of the curves is strongly influenced by the quality factor or inelastic absorption time, respectively. Note that numerical instabilities can strongly influence the theoretical intensity curves at earlier times $(t \leq 21 \mu \mathrm{s})$. Therefore, they were not considered for the diffusion approximation and are not shown in Figs. 9 and 10. For the comparison between experiment and theory, the diffusive time period $41 \leq t \leq 385 \mu$ s from the ultrasound measurements was considered as shown in Fig. 9.

\section{Conclusion}

In summary, the diffusive wave propagation model can be successfully applied to the incoherent wave part of received time signals from ultrasound transmission measurements in watersaturated sintered glass particle packings. A good agreement between time-dependent experimental and theoretical intensity curves could be observed for the (multiply) scattered, incoherent part of the waves. For a sinusoidal input signal of ten cycles with $0.9 \mathrm{MHz}$, the frequency-dependent diffusion coefficient was determined at $D=0.83 \pm 0.03 \mathrm{~m}^{2} / \mathrm{s}$. The transport (diffusive) mean-free path of the ultrasound wave was estimated from the diffusion coefficient as $l^{*}=0.87 \pm 0.03 \mathrm{~mm}$, which corresponds closely to the typical glass bead sizes of $\left\langle d_{p}\right\rangle=1.1 \mathrm{~mm}$ (determined from XRCT data after sintering), which the porous sintered sample is composed of. The effective quality factor, which describes the time-dependent attenuation of the (multiple) scattered wave part, was determined as $Q=190 \pm 10$. Note that this effective quality factor could be used, for instance, in coarsegrained (viscoelastic) continuum models. Therefore, more information about the frequency-dependency of $Q$ would be important, which is out of the scope of this study but necessary for technical applications.

Future work should include the evaluation of the diffusion coefficient and the quality factor of scattered waves for different frequencies and pore fluids to better understand the relevance of the diffusive wave model for acoustical wave propagation in porous systems with $\lambda \approx d_{p}$, which includes also the extension of diffusion models by taking into account the possible occurrence of so-called ultrasound localizations, also known as Anderson localization, as reported in Ref. [42].

We have shown that the diffusion coefficient allows to deduce a mean-free path for scattering events that is very close to the particle size. It would be interesting to examine whether this observation is also valid for different packings with different particles sizes and degrees of polydispersity, for which the mean particle diameter cannot be the only relevant size. Furthermore, it has to be clarified how different sintering treatments and durations affect the scattering behavior of the porous samples and thus the diffusion coefficient and quality factor.

\section{Acknowledgment}

We acknowledge the support from the research program of the Stichting voor Fundamenteel Onderzoek der Materie (FOM).

\section{Funding Data}

- Foundation for Fundamental Research on Matter (Grant No. 09iPOG14-2).

- Nederlandse Organisatie voor Wetenschappelijk Onderzoek (Grant No. 10828).

\section{Nomenclature}

$A(f)=$ complex-valued frequency-dependent amplitude

$c_{e}=$ energy transport velocity

$c_{P 1}=$ sound velocity of P1-wave (coherent wave)

$D=$ diffusion coefficient

$d_{p}=$ glass bead diameter

$E=$ spectral energy density scattered wave field

$E_{0}=$ energy of the transmitted scattered waves

$f=$ frequency

$k=$ wave number

$K_{z}^{s}=$ effective intrinsic permeability in propagation direction $z$

$l^{*}=$ transport mean-free path

$L_{S}=$ sample length

$L_{T}=$ distance between transducer and sample

$N=$ number of speckles

$P=$ spectral energy density of source term

$Q=$ quality factor

$t=$ time

$U(t)=$ voltage of time signal

$V_{p}=$ glass bead volume

$z=$ wave propagation direction

$\sigma=$ dissipation rate

$\tau_{\alpha}=$ inelastic absorption time

$\phi=$ effective porosity

$\omega=$ angular frequency 


\section{References}

[1] Steeb, H., 2010, "Ultrasound Propagation in Cancellous Bone," Arch. Appl Mech., 80(5), pp. 489-502.

[2] Mouraille, O., 2009, "Sound Propagation in Dry Granular Materials: Discrete Element Simulations, Theory, and Experiments," Ph.D. thesis, University of Twente, Enschede, The Netherlands.

[3] Kurzeja, P. S., 2014, "Waves in Partially Saturated Porous Media: An Investigation on Multiple Scales," Ph.D. thesis, Ruhr-University Bochum, Bochum, Germany.

[4] Laugier, P., and Haiat, G., 2011, Bone Quantitative Ultrasound, Springer, Dordrecht, The Netherlands.

[5] Kurzeja, P. S., and Steeb, H., 2012, “About the Transition Frequency in Biot's Theory," J. Acoust. Soc. Am., 131(6), pp. EL454-EL460.

[6] Rempe, M., Mitchell, T., Renner, J., Nippress, S., Ben-Zion, Y., and Rockwell, T., 2013, "Damage and Seismic Velocity Structure of Pulverized Rocks Near the San Andreas Fault," J. Geophys. Res., 118(6), pp. 2813-2831.

[7] Biot, M. A., 1956, "Theory of Propagation of Elastic Waves in a FluidSaturated Porous Solid. I. Low-Frequency Range," J. Acoust. Soc. Am., 28(2), pp. $168-178$.

[8] Biot, M. A., 1956, "Theory of Propagation of Elastic Waves in a FluidSaturated Porous Solid. II. Higher Frequency Range,” J. Acoust. Soc. Am. 28(2), pp. 179-191.

[9] Sheng, P., 1990, Scattering and Localization of Classical Waves in Random Media (World Scientific Series on Directions in Condensed Matter Physics, Vol. 8), World Scientific Publishing, Singapore.

[10] Le Gonidec, Y., and Gibert, D., 2007, "Multiscale Analysis of Waves Reflected by Granular Media: Acoustic Experiments on Glass Beads and Effective Medium Theories," J. Geophys. Res., 112(B5), p. B05103.

[11] Jia, X., 2004, "Codalike Multiple Scattering of Elastic Waves in Dense Granular Media," Phys. Rev. Lett., 93(15), p. 154303.

[12] Griffiths, S., Rescaglio, A., and Melo, F., 2010, "Ultrasound Propagation in Wet and Airless Non-Consolidated Granular Materials," Ultrasonics, 50(2), pp. 139-144.

[13] Güven, I., 2016, "Hydraulical and Acoustical Properties of Porous Sintered Glass Bead Systems: Experiments, Theory, and Simulations," Ph.D. thesis, University of Twente, Enschede, The Netherlands.

[14] Gueven, I., Frijters, S., Harting, J., Luding, S., and Steeb, H., 2017, "Hydraulic Properties of Porous Sintered Glass Bead Systems," Granular Matter, 19(2), p. 28.

[15] Sheng, P., 2006, Introduction to Wave Scattering: Localization and Mesoscopic Phenomena, Vol. 88, Springer-Verlag, Berlin.

[16] Hennino, R., Trégourès, N., Shapiro, N., Margerin, L., Campillo, M., Van Tiggelen, B., and Weaver, R., 2001, "Observation of Equipartition of Seismic Waves," Phys. Rev. Lett., 86(15), p. 3447.

[17] Jia, X., Caroli, C., and Velicky, B., 1999, "Ultrasound Propagation in Externally Stressed Granular Media," Phys. Rev. Lett., 82(9), p. 1863.

[18] Brunet, T., Jia, X., and Mills, P., 2008, "Mechanisms for Acoustic Absorption in Dry and Weakly Wet Granular Media," Phys. Rev. Lett., 101(13), p. 138001.

[19] Weaver, R. L., and Sachse, W., 1995, "Diffusion of Ultrasound in a Glass Bead Slurry," J. Acoust. Soc. Am., 97(4), pp. 2094-2102.

[20] Derode, A., Tourin, A., and Fink, M., 2001, "Random Multiple Scattering of Ultrasound. I. Coherent and Ballistic Waves," Phys. Rev. E, 64(3), p. 036605.

[21] Derode, A., Tourin, A., and Fink, M., 2001, "Random Multiple Scattering of Ultrasound. II. Is Time Reversal a Self-Averaging Process?," Phys. Rev. E, 64(3), p. 036606
[22] Tournat, V., and Gusev, V., 2009, "Nonlinear Effects for Coda-Type Elastic Waves in Stressed Granular Media," Phys. Rev. E, 80(1), p. 011306.

[23] Tournat, V., Castagnède, B., Gusev, V., and Béquin, P., 2003, "SelfDemodulation Acoustic Signatures for Nonlinear Propagation in Glass Beads," C. R. Mec., 331(2), pp. 119-125.

[24] Jia, X., Brunet, T., and Laurent, J., 2011, "Elastic Weakening of a Dense Granular Pack by Acoustic Fluidization: Slipping, Compaction, and Aging," Phys Rev. E, 84(2), p. 020301.

[25] Ji, Q., Le, L., Filipow, L., and Jackson, S., 1998, "Ultrasonic Wave Propagation in Water-Saturated Aluminum Foams," Ultrasonics, 36(6), pp. 759-765.

[26] Lawney, B., and Luding, S., 2014, "Frequency Filtering in Disordered Granular Chains," Acta Mech., 225(8), pp. 2385-2407.

[27] Legland, J., Tournat, V., Dazel, O., Novak, A., and Gusev, V., 2012, "Linear and Nonlinear Biot Waves in a Noncohesive Granular Medium Slab: Transfer Function, Self-Action, Second Harmonic Generation," J. Acoust. Soc. Am., 131(6), pp. 4292-4303.

[28] Shrivastava, R. K., and Luding, S., 2017, "Effect of Disorder on Bulk Sound Wave Speed: A Multiscale Spectral Analysis," Nonlinear Processes Geophys. 24(3), pp. 435-454.

[29] Page, J., Schriemer, H, Jones, I, Sheng, P and Weitz, D., 1997, "Classical Wave Propagation in Strongly Scattering Media," Physica A, 241(1), pp. 64-71.

[30] Weaver, R., 1998, "Ultrasonics in an Aluminum Foam," Ultrasonics, 36(1-5), pp. 435-442.

[31] Page, J. H., 2011, "Ultrasonic Wave Transport in Strongly Scattering Media," Nano Optics and Atomics:Transport of Light and Matter Waves, D. S. Wiersma, R. Kaiser, and L. Fallani, eds., IOS Press, Amsterdam, The Netherlands, pp. 75-93.

[32] Cowan, M., Jones, I., Page, J., and Weitz, D., 2002, "Diffusing Acoustic Wave Spectroscopy," Phys. Rev. E, 65(6), p. 066605.

[33] Becker, J., Jacobs, L. J., and Qu, J., 2003, "Characterization of Cement-Based Materials Using Diffuse Ultrasound," J. Eng. Mech. 129(12), pp. 1478-1484.

[34] Jia, X., Laurent, J., Khidas, Y., and Langlois, V., 2009, "Sound Scattering in Dense Granular Media," Chin. Sci. Bull., 54(23), pp. 4327-4336.

[35] Deroo, F., Kim, J.-Y., Qu, J., Sabra, K., and Jacobs, L. J., 2010, "Detection of Damage in Concrete Using Diffuse Ultrasound," J. Acoust. Soc. Am., 127(6), pp. 3315-3318.

[36] Weaver, R., Zhang, Y., Sachse, W., and Green, K., 1991, "Diffusive Ultrasound Polycrystalline Solids," Ultrasonics International 91, Butterworth-Heinemann, Oxford, UK, pp. 507-510.

[37] Page, J. H., Jones, I. P., Schriemer, H. P., Cowan, M. L., Sheng, P., and Weitz, D. A., 1999, "Diffusive Transport of Acoustic Waves in Strongly Scattering Media," Physica B, 263-264, pp. 37-39.

[38] Viard, N., and Derode, A., 2012, "Experimental Determination of the Diffusion Constant for Ultrasonic Waves in 2-D Multiple Scattering Media With Focused Beamforming," Acoustics, Hong Kong, May 14-18, Paper No. HAL-00810710.

[39] Page, J. H., Schriemer, H. P., Bailey, A. E., and Weitz, D. A., 1995, "Experimental Test of the Diffusion Approximation for Multiply Scattered Sound," Phys. Rev. E, 52(3), pp. 3106-3114.

[40] Carslaw, H., and Jaeger, J., 1986, Conduction of Heat in Solids, Clarendon Press, Oxford, UK.

[41] Job, S., Strybulevych, A., and Page, J. H., 2012, "Ultrasonic Wave Transport in Weakly Confined Granular Media in the Intermediate Frequency Regime," Acoustics, Hong Kong, May 14-18, Paper No. HAL-00811269.

[42] Hu, H., Strybulevych, A., Page, J., Skipetrov, S. E., and van Tiggelen, B. A., 2008, "Localization of Ultrasound in a Three-Dimensional Elastic Network," Nat. Phys., 4(12), pp. 945-948. 